BioMedical Engineering

OnLine

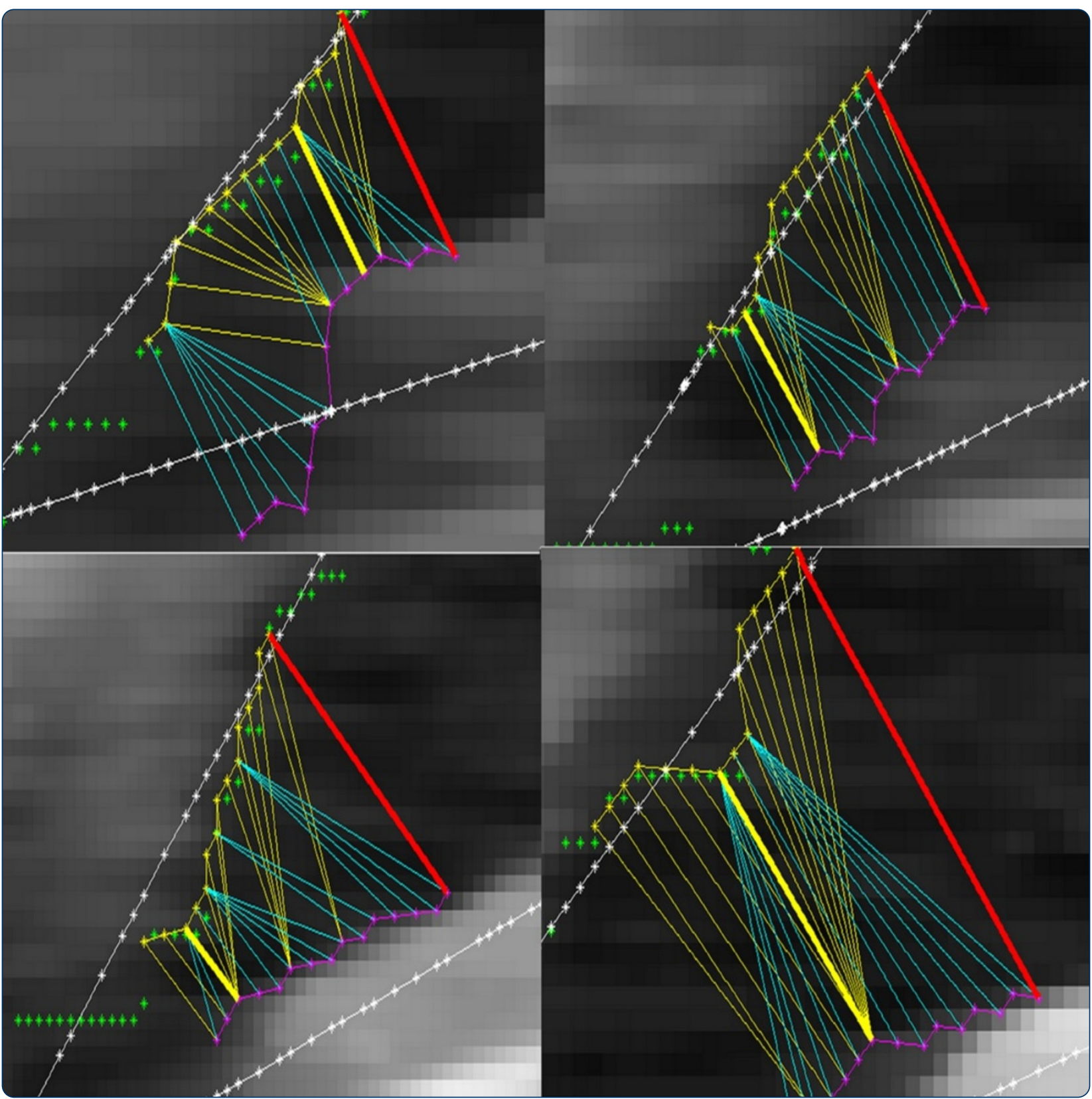

Methods of measuring the iridocorneal angle in tomographic images of the anterior segment of the eye

Koprowski et al. 


\title{
Methods of measuring the iridocorneal angle in tomographic images of the anterior segment of the eye
}

\author{
Robert Koprowski ${ }^{*}$, Zygmunt Wróbel $^{1}$, Sławomir Wilczyński², Anna Nowińska ${ }^{3}$ and Edward Wylęgała ${ }^{3}$
}

\author{
* Correspondence: robert. \\ koprowski@us.edu.pl \\ ${ }^{1}$ Department of Biomedical \\ Computer Systems, Institute of \\ Computer Science, University of \\ Silesia, Będzińska 39 Str, Sosnowiec \\ 41-200, Poland \\ Full list of author information is \\ available at the end of the article
}

\begin{abstract}
Introduction: This paper presents the problem of automatic measurement of the iridocorneal angle in tomographic images of the anterior segment of the eye. It includes the results of the comparison of well-known methods for measuring the iridocorneal angle with new methods, proposed in this paper. All these methods concern tomographic image analysis and processing.
\end{abstract}

Material and method: In total, approximately 100'000 tomographic images (from about 6'000 patients) were analysed. They were obtained using two devices: SOCT Copernicus (Optopol Tech. SA, Zawiercie, Poland) and Visante OCT (Carl Zeiss Meditec, Inc, Dublin, California, USA). The patients, aged 12 to 78 years with varying degrees of the iridocorneal angle pathology, were from the region of Silesia, Poland. The images were in DICOM or RAW formats and analysed in the software developed by the authors for the purposes of this study.

Results: The results indicate that the measurement method proposed by the authors, which is based on the calculation of the minimum distance between the iris and the cornea in the adopted area, is the most accurate. For this method sensitivity was 0.88 , specificity 0.89 and the area under the Receiver Operating Characteristic curve (AUC) was 0.88 . The other known methods for measuring the iridocorneal angle gave worse results, that is, for example, for the measurement of the distance between the iris and the cornea $A U C=0.87$, sensitivity $=0.86$ and specificity $=0.71$. For another well-known method of measuring the iridocorneal angle $A \cup C=0.77$, sensitivity $=0.82$ and specificity $=0.61$.

Conclusions: The study proved that the proposed method of measuring the minimum distance between the iris and the cornea within the adopted area is the most effective in the classification of the iridocorneal angle in patients with a high degree of pathology of all the compared measurement methods based on tomographic images. However, it requires fully automated measurement.

Keywords: Eye, Image processing, Iridocorneal angle, OCT

\section{Introduction}

This paper compares well-known methods for the iridocorneal angle analysis in tomographic images of the anterior segment of the eye with the new ones, suggested by the authors. The iridocorneal angle is the structure responsible for the outflow of aqueous humor from the anterior chamber of the eye. Normal intraocular pressure is determined by the production of aqueous humor by the ciliary epithelium and the rate of humor

\section{() Biomed Central}

(c) 2013 Koprowski et al.; licensee BioMed Central Ltd. This is an Open Access article distributed under the terms of the Creative Commons Attribution License (http://creativecommons.org/licenses/by/2.0), which permits unrestricted use, distribution, and reproduction in any medium, provided the original work is properly cited. 
outflow via two pathways - the trabecular meshwork and the uveoscleral pathway [1,2]. Anatomical anomalies such as the angle narrowing or closure result in impeded outflow and increased intraocular pressure.

The iridocorneal angle is situated on the circumference of the anterior chamber between the sides of the cornea and sclera and the base of the iris and the anterior surface of the ciliary body. Currently, a primary diagnostic tool and technique that enables the analysis of the angle structures is gonioscopy which uses contact gonio lenses [3]. The angle is also studied with OCT devices and the technique is called automatic gonioscopy. The main advantage of this method is its non-invasiveness, whereas the advantage of classical gonioscopy is the ability to visualize pathological structures, such as neovascularization or hyperpigmentation of the weave of the trabecular meshwork [4]. The assessment of the iridocorneal angle using OCT Visante involves morphological assessment and a morphometric analysis of the angle parameters. Morphometric measurements can be carried out using the measuring tool "caliper" included in Visante OCT commercial software. The tool is intended to assist manual operation - Figure 1. An operator typically indicates a point on the scleral spur in a OCT image (marked with a white circle in Figure 1) whereas a device adjusts the position of the other points (marked with red circles - in Figure 1). In order to perform these morphometric measurements, it is necessary to manually locate the scleral spur, which is a landmark for determining morphometric measurements, in the scan "ASS". In practice, it often happens that the scleral spur is not visible. According to the results given in paper [5], such a situation occurs in approximately $20 \%$ of cases. The known methods TIA, AOD 500, AOD 750, TISA 500, TISA 750 can be defined on the basis of these morphometric measurements - Figure $2[6]$ and Table 1.

These methods are described in detail in papers [1-3,7]. The values "500" and "750" refer to the distance, expressed in microns, from the scleral spur. As mentioned above, on the basis of the scleral spur location point indicated by an operator, the other characteristic points are drawn automatically. They are necessary to perform calculations for the presented methods, namely TIA, TISA and AOD. Undoubtedly, this semiautomatic method facilitates operator's work but still it is not done fully automatically. Fully automatic measurement was suggested by the authors in 2011 [7]. It involves the use of information about iridocorneal contours. Each iris contour point is combined
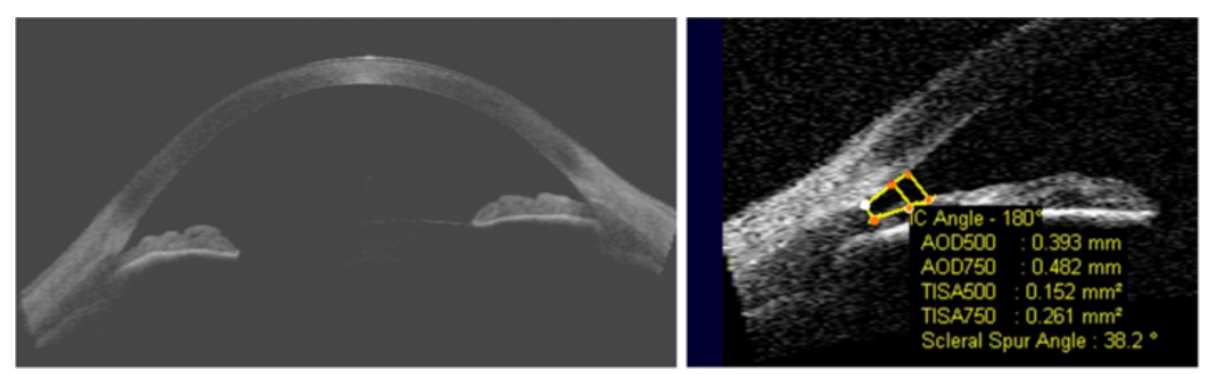

Figure 1 Tomographic image of the anterior segment of the eye and examples of commercial software operation provided with the Visante OCT device. In the illustrated case, an operator indicates one point for the scleral spur position (highlighted with a white circle) and the machine adjusts its position and draws the location of consecutive points marked with red circles. The results for the analysed case are $A O D=393 \mu \mathrm{m}, T I S A=0.152 \mathrm{~mm}^{2}, T I A=38.2^{\circ}$. 

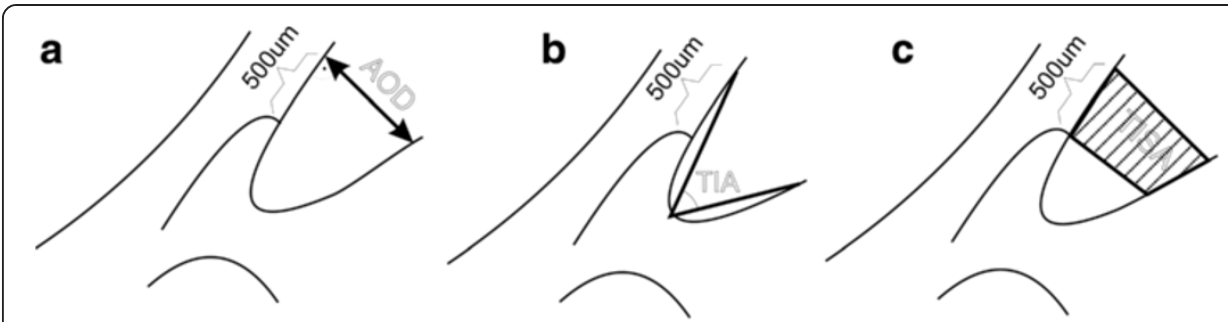

Figure 2 Methods of measuring the iridocorneal angle. a) AOD 500 (Angel Opening Distance) involves measuring a distance between a point of the cornea which is $500 \mu \mathrm{m}$ away from the scleral spur and the opposite point of the iris. b) TIA (Trabecular-Iris Angle) involves a direct measurement of the angle. c) TISA 500 (Trabecular-Iris Space Area) involves measuring an area covering $500 \mu \mathrm{m}$ located in the area bounded by the cornea and the iris.

with a suitable corneal contour point. A division of contours is performed based on the point of the greatest curvature (point marked in black, the starting point of the coordinate system - Figure 3). In this way, a sequence of measurements at various distances from the apex of the measured angle is obtained - the chart shown in Figure 3. This method, referred to as $A O S$ (Angle Opening Sequence), ensures obtaining much more information on the iridocorneal angle when compared to AOD, TIA and TISA. The varying degree of the iridocorneal angle pathology visible in Figure 4 (especially narrow or closed iridocorneal angle), which is difficult to measure with conventional methods such as TIA, TISA and AOD, is successfully and reliably evaluated using AOS [8]. It is apparent from the examples shown in Figure 4 that a difficulty in a reliable assessment of the iridocorneal angle with the AOD, TIA and TISA methods lies primarily in a large extent of pathology - distorted sides of the angle, especially of the iris angle. Therefore, in pathological conditions, measurement results of TISA and $A O D$ are strictly dependent on measurement locations. Slightly less sensitive to this type of pathology is the TISA method. However, its practical application is limited due to the area unit $\left(\mu \mathrm{m}^{2}\right)$ which is less intuitive and difficult to quickly compare with the other methods, $T I A$ and $A O D$. In practice, the described methods for measuring the iridocorneal angle, namely TIA, TISA and $A O D$, have a number of inconsistencies and irregularities in the interpretation of results. As a consequence, results are not repeatable, reliable and difficult to verify and compare with the model and other doctors' results. The situation becomes critical when the progress of treatment or the disease progression of a patient diagnosed by different doctors in different medical centres equipped with different types of OCT devices needs to be assessed.

Archiving results is an important issue in practical measurements. In the case of TIA, TISA and $A O D$, each measurement is connected with archiving one scalar value, e.g. the angle value measured with TIA with a typical accuracy of one decimal place. In the

Table 1 Known methods for measuring the iridocorneal angle and their definitions

\begin{tabular}{lll}
\hline Method symbol & Method name & Definition \\
\hline AOD & Angle Opening Distance & $\begin{array}{l}\text { (Figure 2a) involves measuring a distance between a point } \\
\text { of the cornea which is } 500 \mu \mathrm{m} \text { away from the scleral spur } \\
\text { and the opposite point of the iris }\end{array}$ \\
TIA & $\begin{array}{l}\text { Trabecular-Iris Angle } \\
\text { TISA }\end{array}$ & $\begin{array}{l}\text { (Figure 2b) involves a direct measurement of the angle } \\
\text { (Figure 2c) involves measuring an area covering } 500 \mu \mathrm{m} \\
\text { located in the area bounded by the cornea and the iris }\end{array}$ \\
\hline
\end{tabular}




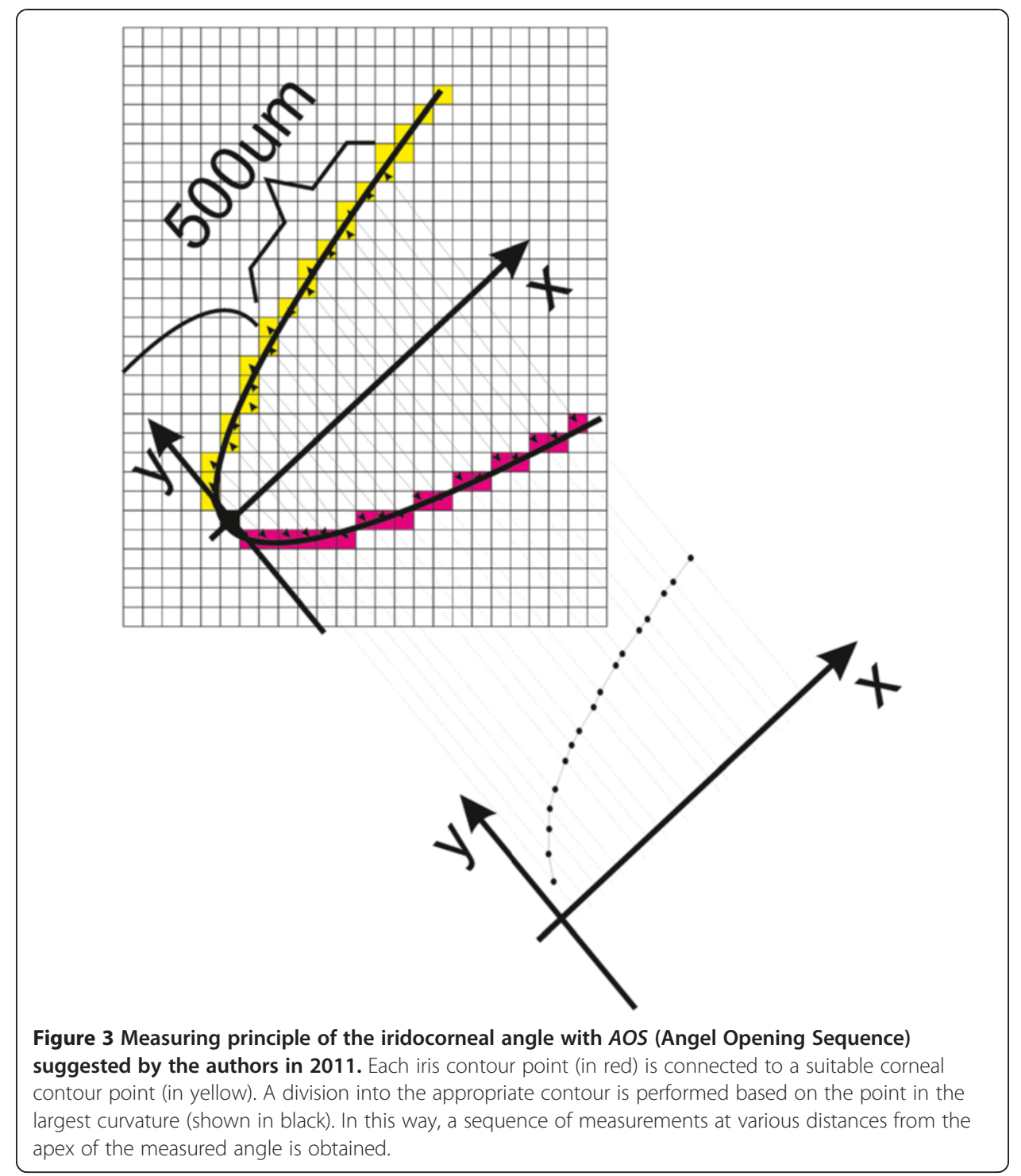

case of $A O S$, a data vector of successive measurements of iridocorneal contour distances is archived. In paper [7] in 2011, the authors presented a new way of recording the results obtained with the AOS method, which included the alphabet shown in Table 2.

The alphabet has been adopted in clinical practice. However, its practical application is somewhat troublesome due to more complicated recording in comparison to

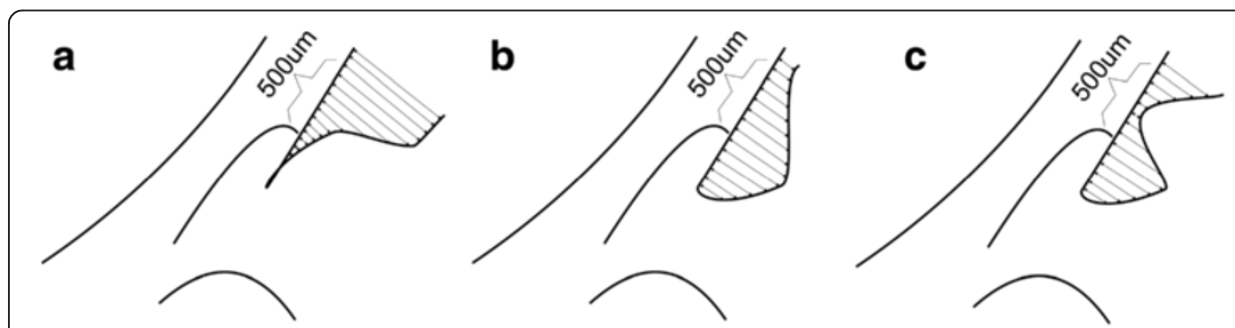

Figure 4 Various degrees of the iridocorneal angle pathology with marked measurement points for AOS. The presented cases $\mathbf{a}), \mathbf{b}$ ) and $\mathbf{c}$ ) are not correctly measured with TIA, TISA and AOD. This is due to both a visible pathology (a variable iris shape) as well as the definition of TIA, TISA and AOD. 
Table 2 Alphabet for the iridocorneal angle description proposed by the authors in 2011

\begin{tabular}{ll}
\hline Symbols & Function \\
\hline $\mathbf{v}$ & increasing distance for successive values on the axis 0x (Figure 3) \\
- & local minimum \\
& local maximum \\
& constant distance value for increasing values of $0 x$ \\
& Numerical parameters \\
& angular value, \\
& maximum, minimum or fixed distance for specific 0x \\
& range of values on the axis $0 x$ in which a given situation occurs \\
\end{tabular}

conventional, previously known, methods (TIA, TISA, AOD). As a result, medical errors are more likely due to improper recording. Therefore, the authors suggested a new method, modified in relation to $A O S$, for measuring the iridocorneal angle, namely $A O M$ (Angel Opening Minimum) which is described below.

\section{Material}

In the study, about 100'000 tomographic images (from about 6'000 patients) were examined. The images were acquired using the following devices: SOCT Copernicus (Optopol Tech. SA, Zawiercie, Poland) and Visante OCT (Carl Zeiss Meditec, Inc, Dublin, California, USA). The patients, aged 12 to 78 years with varying degrees of the iridocorneal angle pathology, were from the region of Silesia, Poland. The obtained images were in DICOM or RAW formats with a resolution of $256 \times 1024$ pixels, within a measuring range of $8 \mathrm{~mm} \times 16 \mathrm{~mm}$, which gives $31.3 \mu \mathrm{m} /$ pixel. The image analysis was carried out in a Matlab software package with Image and Signal Processing toolboxes and the code was optimized in the $\mathrm{C}$ programming language.

\section{AOM method}

The $A O M$ (Angle Opening Minimum) method involves determining a minimum distance, which is one scalar value, between the contours of the iris and cornea. To be more exact, it is the distance between different points of the cornea and the nearest point of the iris. The measured area of the cornea covers a range of $500 \mu \mathrm{m}$ or $750 \mu \mathrm{m}$ starting from the scleral spur. Details on the principle of the AOM method of measurement are shown in Figure 5a. Figure 5b, on the other hand, shows an opposite situation, where a minimum distance between various points of the iris and the nearest point of the cornea is calculated - the AOM2 method. The AOM method is based on a sequence of calculations of the minimum angle values for individual pixels of the cornea edge from all the pixels of the iris edge (or the other way round in AOM2 - Figure 5). Measurements can be carried out in the entire range for each of the contour points of the iris or cornea (as in Figure 5c and 5d) but only the contour points which are realized in the range of $500 \mu \mathrm{m}$ of the corneal contour are relevant. This narrowing of the measurement range usually takes place in the last stage of calculations.

The obtained results differ depending on the measurement method (AOS, AOM or AOM2 - Table 3). Examples of a sequence of distance measurements are shown in Figure 6. The graph shows changes in values of the angle opening for successive pixels of 

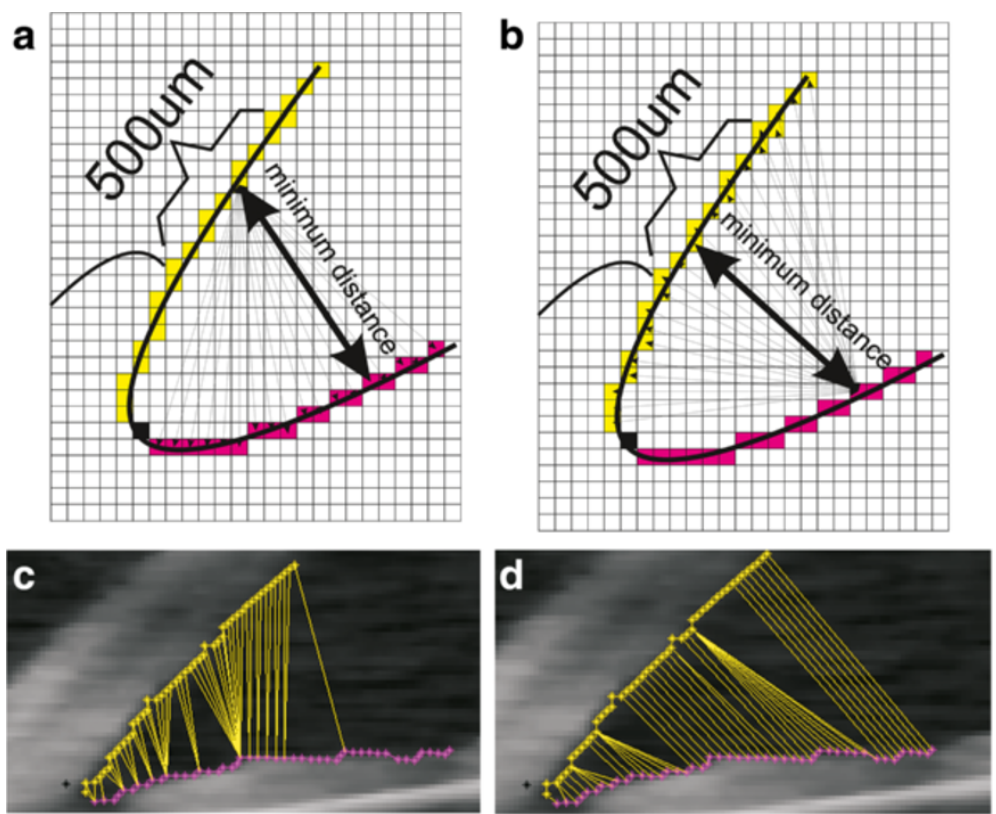

Figure 5 The conception of $A O M$ (Angle Opening Minimum) and AOM2 methods and the obtained results. a) shows how the shortest distance between all the points of the cornea and one of the sample points of the iris is chosen in the AOM method. The shortest distance for both variants for one analysed pixel is highlighted in bold arrow. b) shows how the shortest distance between all the points of the iris and one of the sample points of the cornea is chosen in the AOM2 method. c) and $\mathbf{d}$ ) show practically obtained results for all pixels in the implementation of the two variants: the AOM method shown in a) and the AOM2 method shown in b). In order to better visualize the results, the analysis was not limited to the appropriate range of $500 \mu \mathrm{m}$.

the contour of the cornea or iris, respectively, for the images in Figure $5 \mathrm{c}$ and $5 \mathrm{~d}$. The largest visible difference is between the $A O M$ method and the other methods, namely AOM2 and AOS. The difference is due to different measurement methods and amounts to approximately 10 pixels $(10$ pixels * $31.3 \mu \mathrm{m} / \mathrm{pixel}=313 \mu \mathrm{m})$ in the range of $500 \mu \mathrm{m}$ which is marked in yellow (Figure 6). In practice, due to a greatly variable shape of the contour of the iris in comparison with the contour of the cornea, the AOM method produces underestimated measurement values. However, they are more reliable when compared to the AOS or AOM2 methods. They enable to take into account the iridocorneal angle pathology to a greater extent. Such situations concern the narrowing of the iridocorneal angle in the area outside the range of $500 \mu \mathrm{m}$ when accurate (consistent with the definition) calculations with the methods TISA, TIA and AOD do not produce satisfactory results.

The result of the measurement of the iridocorneal angle with the $A O M$ method is therefore a minimum distance between the iris and the cornea measured in the area of

Table 3 Methods for measuring the iridocorneal angle proposed by the authors and their definitions

\begin{tabular}{lll}
\hline Method symbol & Method name & Definition \\
\hline AOS & Angle Opening Sequence & $\begin{array}{l}\text { (Figure 3) each iris contour point is combined with a suitable } \\
\text { corneal contour point } \\
\text { AOM }\end{array}$ \\
AOMgle Opening Minimum & $\begin{array}{l}\text { (Figure 5a) shortest distance between all the points of the } \\
\text { cornea and selected range of the iris }\end{array}$ \\
& Angle Opening Minimum 2 & $\begin{array}{l}\text { (Figure 5b) shortest distance between all the points of the } \\
\text { iris and selected range of the cornea }\end{array}$ \\
\hline
\end{tabular}




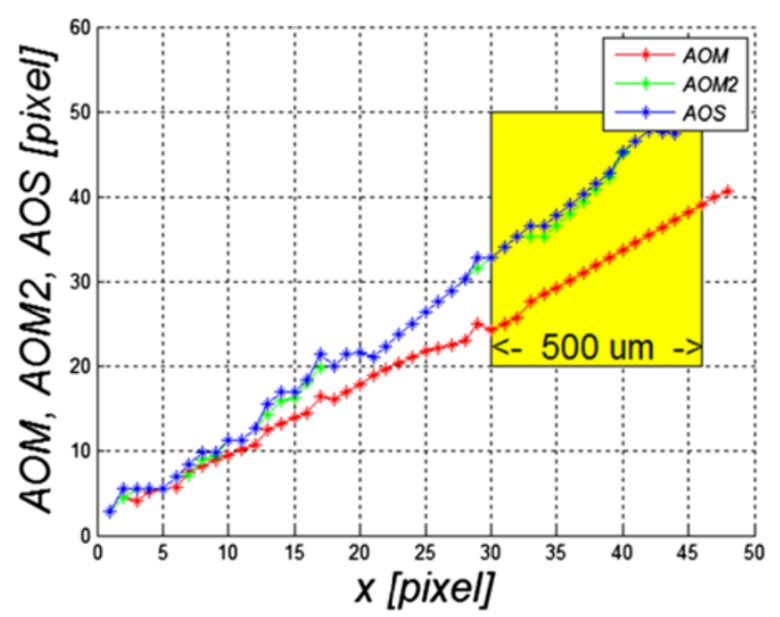

Figure 6 Graph of changes in values of the angle opening for successive pixels of the contour of the cornea or iris, respectively (depending on the measurement method) shown in Figure $5 \mathrm{c}$ ) and d). The graph shows the biggest difference between the AOM method and the other methods (AOM2 and AOS). Visible differences are due to different measurement methods described in the paper. The area highlighted in yellow includes the range of $500 \mu \mathrm{m}$ measured from the scleral spur ( 16 pixels $\cdot 31.3 \mu \mathrm{m} / \mathrm{pixel} \cong 500 \mu \mathrm{m}$ ).

$500 \mu \mathrm{m}$ starting from the scleral spur. The above mentioned range of $500 \mu \mathrm{m}$ of the corneal contour (16 pixels fall into this range for the analysed image resolution) and all the pixels in the contour of the iris are taken into account in the measurements.

\section{Implementation of the AOM method}

The $A O M$ method requires full automation of image analysis. This automation enables to detect the cornea and iris edges and then perform adequate calculations of the minima (according to the methodology of $A O M$ described above). The image analysis algorithm, in particular, should include the following elements [9-12] shown in Figure 7. The need to use a profiled algorithm in this case is connected with inadequate results obtained with other known algorithms for detecting lines and/or areas in the image.

- Hough's transform [7] enables to detect lines in images of a pre-selected shape. However, the results in the case of large inter-individual variability are not satisfactory.

- wavelet analysis method [13] gives incorrect results when objects are hard to see and the lines overlap - such situations are quite common in the case of the analysed images,

- analysis methods of elongated objects cannot be applied here due to a possibility of large changes in the size of both the object itself and its thickness and a possibility of its division into multiple parts (e.g. the iris or cornea).

- object recognition methods in cases of large pathology can give unpredictable results.

- other known methods, for example, texture analysis [14], also do not produce satisfactory results.

Based on this and the literature review [8,15-18] and given the medical evidence presented below, a profiled algorithm for the analysis and processing of images of the front part of the eye was suggested. 


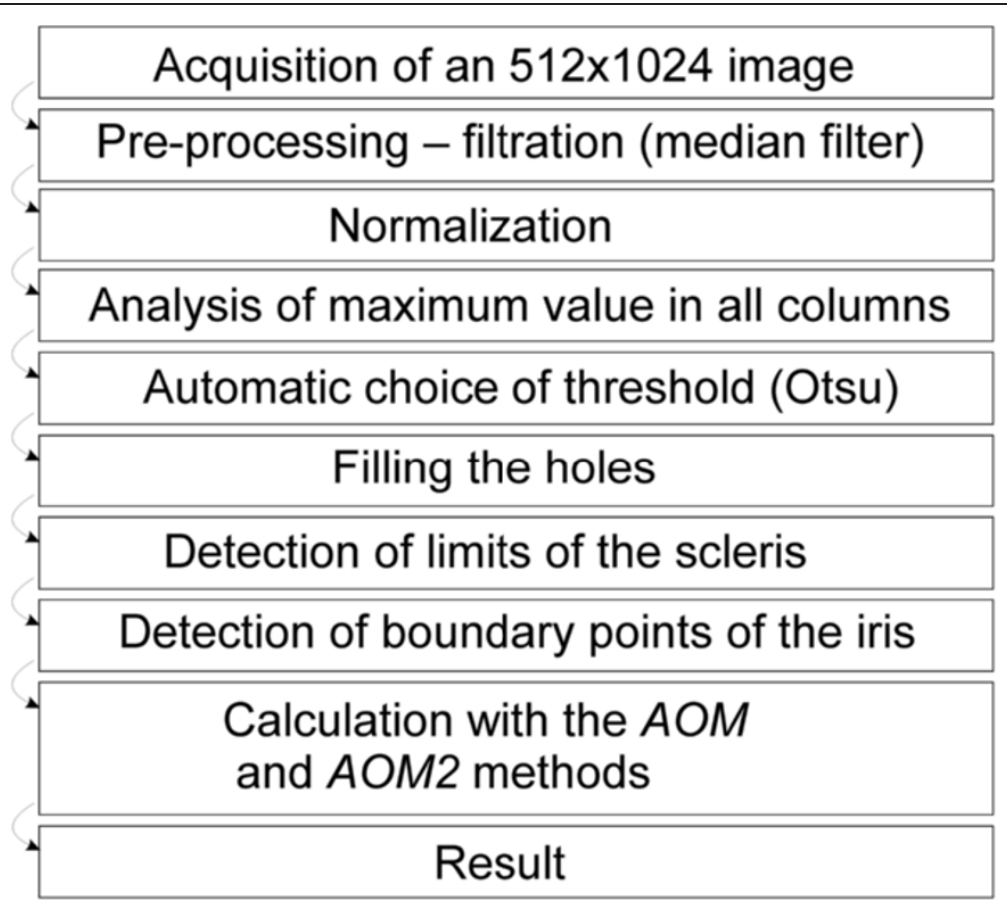

Figure 7 Block diagram of the tomographic image analysis algorithm in the $A O M$ method. The presented algorithm enables fully automatic measurement of the iridocorneal angle. The obtained result is in the form of one scalar value which is the minimum distance between the selected ranges of the iris and cornea. The presented algorithm is versatile and provides correct results for any tomographic images of the eye.

In the implemented algorithm, an input image with the resolution of $256 \times 1024$ pixels, mentioned in the introduction, is entered into the developed software in DICOM format. Then filtration with a median filter (with a $3 \times 3$ pixel mask) is carried out followed by the analysis of each column. As a result of this analysis, a binarization threshold is calculated for each column (Otsu method [19]). The created binary image is shown in Figure 8b). In the next stage, the method of filling the holes is applied in
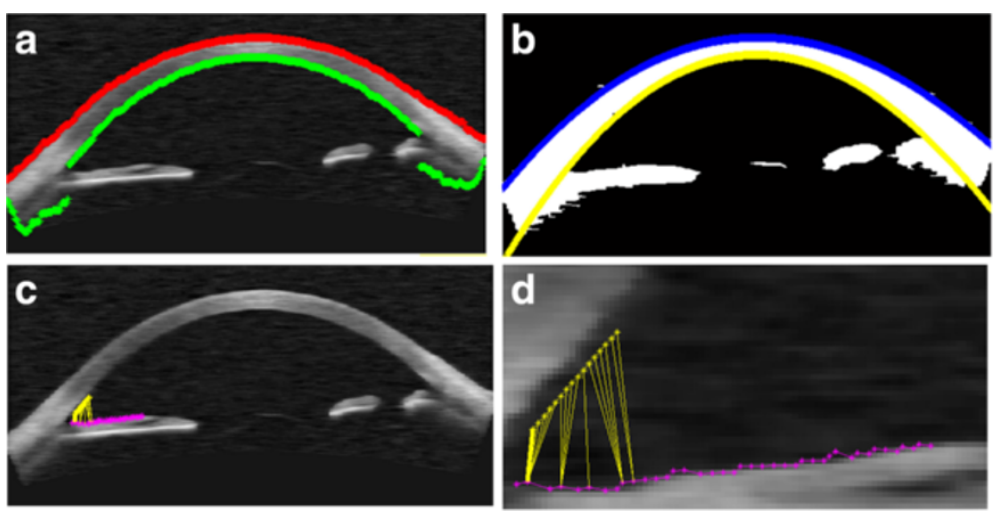

Figure 8 Some stages of processing in the calculation of the iridocorneal angle with the AOM method: a) the sclera and cornea boundaries (in green and red) set automatically, b) approximation of the automatically set sclera boundary - in blue and yellow, c) the iridocorneal angle set automatically with the $A O M$ method and its enlargement d). According to the definition, the iridocorneal angle calculated with the proposed $A O M$ method is a minimum distance calculated between the cornea pixels (the area of $500 \mu \mathrm{m}$ ) and all the pixels of the iris boundary. It is marked with a bold yellow line - d). 
order to eliminate minor inclusions or detachment. In this pre-prepared image, the sclera boundaries are determined followed by approximation of the boundaries with a polynomial of degree 4 (Figure $8 \mathrm{~b}$ ). After the analyses of the iris, ciliary appendages and scleral spur, there follows the analysis of iris termination points which uses information from the inside of the sclera boundary (Figure 8c). At this stage, the iridocorneal angle can be determined using the TIA, TISA and $A O D$ methods. The value of the iridocorneal angle measured with the $A O M$ method (or $A O M 2$ ) requires a calculation of Euclidean distance for each possible pair of points (iris-cornea). The result is a minimum distance between them calculated in the range of $500 \mu \mathrm{m}$, highlighted in Figure $8 \mathrm{~d}$ in bold yellow.

This algorithm correctly identifies and calculates the iridocorneal angle not only with the AOM method but also with the other methods, namely AOS, AOM2 or TIA, TISA and $A O D$ at 500 and $750 \mu \mathrm{m}$ from the scleral spur. All the algorithm parameters are calculated automatically. The algorithm automatically adjusts to the type and brightness of a derived image (pre-processing, filtering with a median filter with a $5 \times 5$ pixel mask or normalization). During the programme installation, an operator only gives a distance attributable to the pixel (strictly dependent on the type of the used tomographic camera) if it is not specified in the DICOM header. This information is necessary to calibrate the measured values of the iridocorneal angle (in the analysed images obtained with Visante OCT it is $31.3 \mu \mathrm{m} / \mathrm{pixel}$ ).

Accuracy of the measurements and the comparison of the results obtained with the above methods (AOD, TIA, TISA, AOS, AOM and AOM2) are presented below.

\section{Comparison of $A O D, T I A, T I S A, A O S, A O M$ and $A O M 2$ methods in practice}

A practical application of the measurement methods, namely AOD, TIA, TISA, AOS, $A O M$ and $A O M 2$, requires designation of the contours or a single point of the cornea and iris. In addition, it is necessary to determine the scleral spur location. The currently available software for OCT devices does not enable the mentioned fully automatic measurement. Calculations are carried out either manually or semi-automatically. For this reason, the results are not repeatable - they depend on an individual choice of the scleral spur (iridocorneal angle) location by an operator. The presented algorithm enables fully automatic measurement with the above mentioned methods. Therefore, their comparison is possible.

Of all the mentioned methods ( $A O D$, TIA, TISA, AOS, AOM and AOM2), only AOD, TIA, AOM2 and AOM are further taken into account. The TISA method is rarely used in practice due to the unit of the iridocorneal angle, that is, $\mu \mathrm{m}^{2}\left(\mathrm{~mm}^{2}\right)$. The AOS method is also difficult to compare with other results because of a difficulty in comparison of an $A O S$ data sequence (or the mentioned alphabet) with scalar values obtained as results from other methods.

The other methods, $A O D$, TIA, AOM2 and $A O M$, can be compared using one of two ways. One of them uses a model (an artificial image) with a known and measured iridocorneal angle and the other one compares results with those obtained by ophthalmology experts. The first way, which will not be used here, is to use an artificial image containing known values of the iridocorneal angle realized with resolution error precision at the time of creating the image. The other way is to use the results obtained from an assessment performed by an ophthalmology expert or from other more 
accurate measurement methods (if they exist). Both ways have their advantages and disadvantages. The disadvantage of the first method is a difficulty in close conjunction of artificial images with real images which reflect a full range of variability. Its advantage is an ability to quickly compare the results and no need for the presence of an ophthalmologist. In the other method, results are obtained from a real image, but it requires a tedious procedure that involves manual marking (by an operator) of individual values of $A O D$, TIA, AOM2 and AOM. This method is also highly dependent on the observer's subjective judgment which is mainly related to the determination of significance of details in an image.

As a result, the comparison of the methods $A O D$, TIA, AOM2 and $A O M$ was carried out in two stages.

\section{Comparison with the results obtained by an expert}

In the first stage, an ophthalmologist manually marked the iridocorneal angle for the first 100 images with the $A O D$, TIA, AOM2 and AOM methods. In this case, a measurement error $\delta_{q}$ was calculated. It was defined as:

$$
\delta_{q}=\frac{w_{M}-w_{P}}{w_{P}} .100 \%
$$

where:

$w_{M}$ - the measured value,

$w_{P}$ - a correct value measured with more accurate methods (e.g. manually) or an average value of a series of measurements,

$q$ - an index indicating the measurement method: $A O D$, TIA, AOM2 or AOM.

The obtained values of $\delta_{q}$ are not greater than $5 \%$ for the $A O D$ and TIA methods $\left(\delta_{A O D}=4.5 \%, \delta_{T I A}=4.8 \%\right)$ and for $A O M$ and $A O M 2$, they are $\delta_{A O M}=7.2 \%$ and $\delta_{A O M 2}=$ 9\%, respectively. Therefore, it seems that the presented $A O M$ and $A O M 2$ methods are worse than the known methods. The reason for larger error values for these methods is the principle of measurement. It is much harder to manually select iris contour points located closest to the analysed corneal contour pixel (Figure 5) and then determine, of all the points, which of them is the smallest. Thus, it is necessary to compare the methods in terms of reliability of the iridocorneal angle calculation and detection of pathological cases. This methodology is described below.

\section{Comparison with the results obtained with other known methods}

In the second stage, the comparison of the AOD, TIA, AOM and AOM2 methods for all the obtained images (approximately 100'000) was performed by standardization of the measurement unit to relative values. This comparison was related to the effectiveness of detection of cases with normal and narrow iridocorneal angle, including pathological conditions. In order to compare the results, the following typical measures were used: accuracy $A C C=(T P+T N) /(T P+T N+F P+F N)$, specificity $S P C=T N /(F P+T N)$ and sensitivity $T P R=T P /(T P+F N)$ where: $T P$ - true positive, $T N$ - true negative, $F N$ - false negative and $F P$ - false positive. These measures were used to evaluate the $A O D$, TIA, $A O M 2$ and $A O M$ methods.

All cases where the iris or cornea was not visible were pre-eliminated. Such situations occurred when OCT imaging was not focused on calculating the iridocorneal angle. 
After removing such cases, 83'574 images remained for further analysis. It was carried out in the steps presented below.

\section{Removal of all atypical cases for which the algorithm designated too short or too long} iris edge

Atypical cases are those for which the iris edge designation contains less than 16 pixels (500 $\mu \mathrm{m}$, Figure $9 \mathrm{a}$ - in blue) or more than 70 to 80 pixels, which is equivalent to $2.5 \mathrm{~mm}$ (Figure 9a - in yellow). These limitations are natural and result from the presented methodology of measurement of AOD, TIA, AOM2 and AOM. The distance from the scleral spur boundary used in the measurement is $500 \mu \mathrm{m}$, which corresponds to a minimum of 16 pixels. This limitation (16 pixels) arises only from the need to compare these methods, and smaller values $(<16)$ do not indicate errors in the algorithm operation. There are 48'022 images of this type whose length of the iris contour is below 16 pixels. This represents $57.5 \%$ of the analysed images. Values above approximately 70 to 80 pixels of the iris edge length do not occur in medicine; therefore, they indicate an error in the algorithm operation. As is apparent from the graph showing the iris contour length for individual images, a number of such cases (exceeding 70 pixels) is relatively low (3'458, which is $4.1 \%$ of all cases). In total, 32 '094 images remained for further analysis after applying these two limitations.

\section{Comparison of the results obtained for the proposed AOM method and the AOD method}

For this purpose, $\triangle_{A O D, A O M}$ was calculated as a difference between $A O D$ and $A O M$. The results are shown in Figure 9b. The graph (Figure 9b) was divided in two parts. The changes in the value of $\triangle_{A O D, A O M}$ highlighted in green when compared to the $A O M$ and $A O D$ methods indicate undervaluation of the iridocorneal angle measurement for the $A O M$ method. This situation is consistent with the definition according to which in the case of the $A O M$ method, it is the smallest distance between the corresponding points in the iris and cornea that is sought. The part of the graph marked in yellow (Figure 9b) covers a range of pathological cases for which a direct comparison

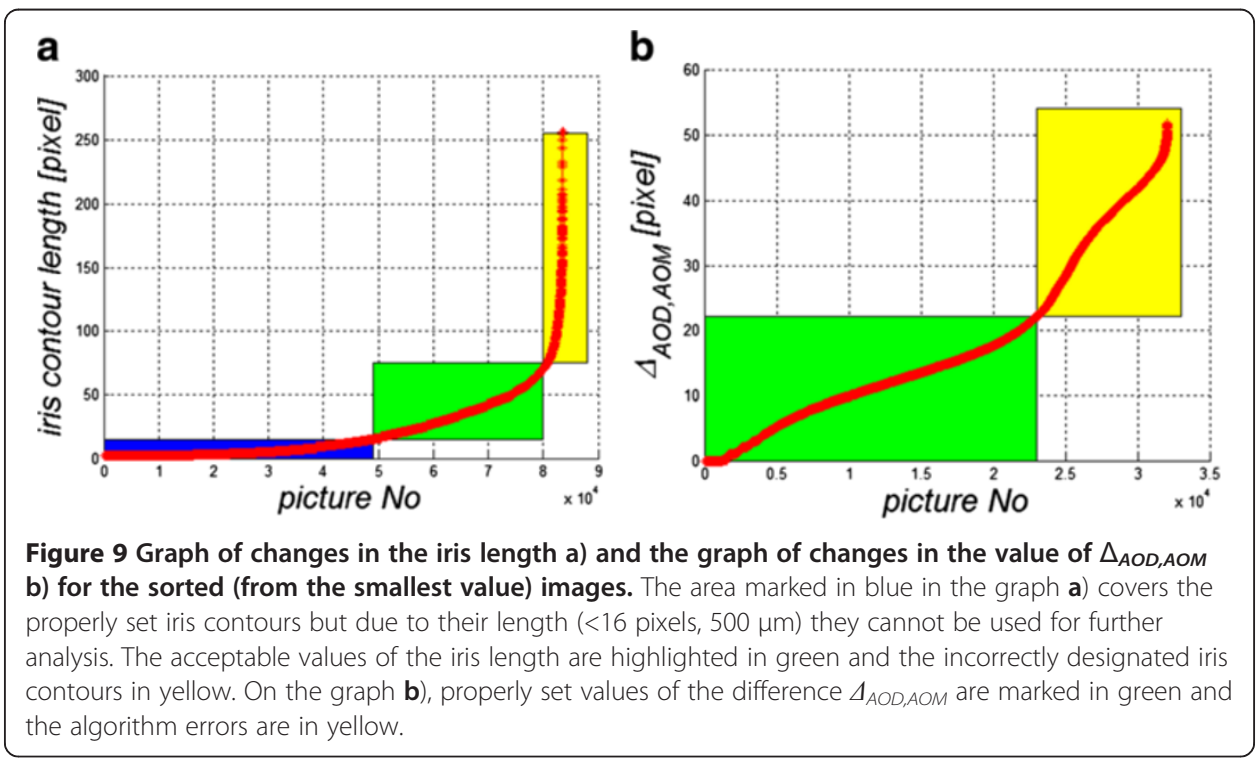


of $A O M$ and $A O D$ was not possible (incorrect location of the area of analysis for the $A O D$ method or incorrect indication of a minimum in the $A O M$ method). The graph of $\triangle_{A O D, A O M}$ as a function of a sequence of images was divided in two parts (Figure 9b):

- with differences between $A O D$ and $A O M$ arising from their definition (in practice to about 22 pixels) and,

- with differences between $A O D$ and $A O M$ caused by the algorithm errors.

The division between the two areas (the threshold value of 22 pixels) was verified in practice and included a manual analysis of borderline cases. This value also means that in carrying out a calculation for the afore mentioned group of images, the difference in the results for $A O D$ and $A O M$ methods will not be greater than 22 pixels for the most obtuse iridocorneal angle.

As it turns out, in practice, the $A O M$ method enables a proper evaluation of specific cases of the iridocorneal angle. These examples are shown in Figure 10. Thus it is possible to obtain correct classification of images with a proper, open iridocorneal angle from pathological cases with the risk of iridocorneal angle closure.
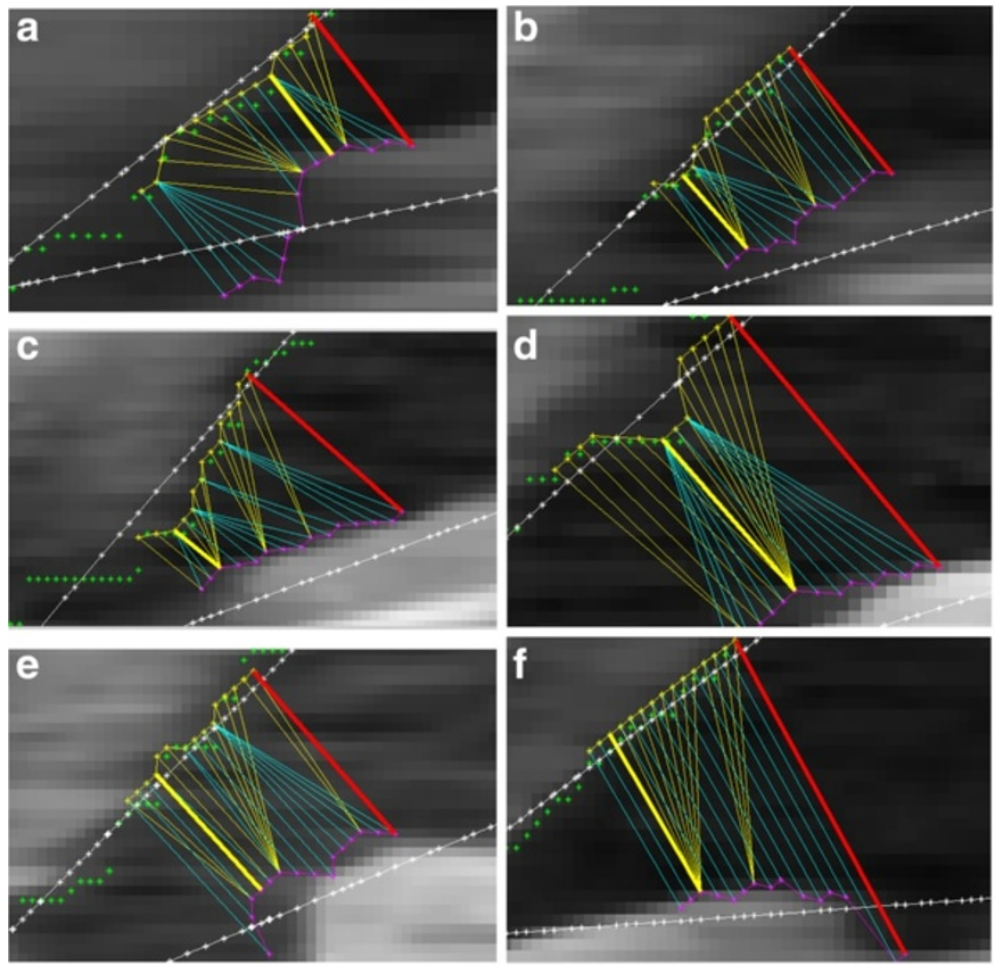

Figure 10 Sample image fragments of the iridocorneal angle for which the known $A O D, T I A$ and TISA methods give incorrect results. For these examples, the iridocorneal angle is determined properly with $A O M$ and $A O D$ methods. The results obtained with the $A O M$ method are marked with a bold yellow line, and the results obtained for the $A O D$ method are in red. The other coloured lines refer to the cornea and iris contours (yellow, magenta), and sides of the angle of the TIA method (sides are shown as a linear interpolation of the iris and cornea contours) are marked in white. The results obtained for the examples are: a) $A O D=12.9, A O M=7.8, T I A=29.7$ b) $A O D=15.6, A O M=9.2, T I A=27.1$ c) $A O D=22.6, A O M=6.5, T I A=29$ d) $A O D=26, A O M=16, T I A=24.5$ e) $A O D=21.6, A O M=15.9, T I A=22.6$ f $A O D=30.2, A O M=15.2, T I A=29.8$ ( $A O M$ and $A O D$ values are given in pixels, $T I A$ values in degrees). 


\section{Comparison of quality of classification for the methods AOM, TIA and AOD}

This comparison was made for a classification of healthy subjects and patients with the risk of iridocorneal angle closure $\left(T I A<15^{\circ}, A O D<190 \mu \mathrm{m}, T I S A<0.11 \mathrm{~mm}^{2}[5,20]\right)$. Model values of the classification (due to the size of the group) were determined as the average of measurements performed with the AOS and TISA methods; additionally, in some cases, as the average of the results corrected by an expert. The division gave 20'536 cases with a correct iridocorneal angle and 2'454 cases with an incorrect narrowed angle. The $A O M, T I A$ and $A O D$ methods were compared for which the ROC (Receiver Operating Characteristic) curves shown in Figure 11 were obtained. For each method, the cut-off threshold was changed in the range from 0 to 180 by every 1 , covering both the range of angular values (in the case of TIA) and the range of distance changes given in pixels $(A O M$ and $A O D)$. For the $T I A$ method, optimal values were obtained for the threshold $T I A=18^{\circ}$, i.e.: $T P R=0.82$ and $S P C=0.61$. These are the worst results obtained for the compared methods. In this case, AUC is 0.77 . The other compared method is $A O D$. In the case of this method, an optimal cut-off threshold is $A O D=8$ pixels (about $240 \mu \mathrm{m}$ ). With this threshold value, $T P R=0.86$ and $S P C=0.71$ and $A U C$ is 0.87 . The last compared method is the new $A O M$ method. It gave the best results for a threshold cut-off $A O M=6$ pixels (about $180 \mu \mathrm{m}$ ) where $T P R=0.88, S P C=$ 0.89 and $A U C=0.88$.

The adopted measurement definitions result from differences between the results. Distance point measurements are the main reason for errors in $A O D$. This method is very sensitive to noise and artefacts in images which can be observed especially in cases of high degrees of pathology. For the TIA method the situation is similar. A fixed point position of the angle measurement is very sensitive to noise. Another difficulty lies in finding an appropriate point of the angle apex [5,21,22]. Perhaps arranging the angle

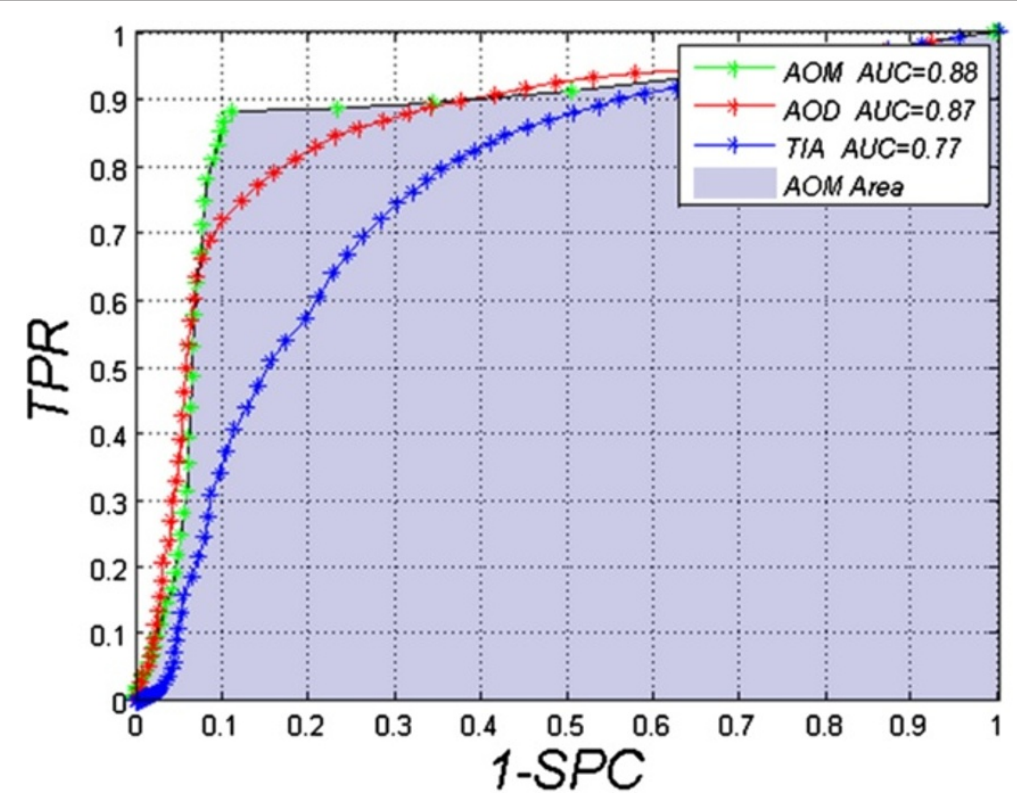

Figure 11 ROC (Receiver Operating Characteristic) graph of the evaluation of classification for TIA, $A O D$ and $A O M$. The area under the curve for the best classifier of $A O M$, i.e. $A \cup C=0.88$ is marked in blue. The other methods produce worse results i.e.: for $A O D, A \cup C=0.87$ and for $T I A, A \cup C=0.77$. Differences in the obtained classification quality result from the conception of measurement with TIA and AOD methods. 
sides as a line approximating the iris and cornea contours would produce better results. The TISA method seems to be the most appropriate here. However, local narrowing of the iridocorneal angle enables to obtain the same results as in the case of slight angle narrowing, but in a larger area $(500 \mu \mathrm{m})[23-26]$.

\section{Conclusions}

The paper presents the comparison of known methods and proposes a new method of measuring the iridocorneal angle on the basis of tomographic images. This comparison shows that the best method is the one presented by the authors, namely $A O M$ for which $A U C=0.88$. This result is by 0.01 better than that obtained for the well-known $A O D$ method (Tables $1,2,3)$. In terms of specificity, the difference between $A O D$ and $A O M$ is even greater (Figure 11) and amounts to 0.18. The differences between $A O D$ and $A O M$ result from specificity of measurement. For example, measurements for the $A O D$ method (as well as for another method, i.e. TIA) are carried out pointwise [27-29]. The whole area of the iridocorneal angle, i.e. in the range of $500 \mu \mathrm{m}$ starting from the scleral spur [5], is not taken into account. Moreover, minimum distance values are not calculated unlike in the $A O M$ method. $A O M$ enables to obtain reliable results in the form of a single scalar value in comparison with $A O S$ (for which a sequence of values is obtained). Although AOS enables visualization of more complex cases (of the iridocorneal angle calculated for large pathology) in the form of a graph, it requires recording a series of numbers (which refer to distances between the retina and the cornea). One of the disadvantages of the $A O M$ method is the need to perform calculations (of minimum distance) on a computer. Manual calculations of the iridocorneal angle with the $A O M$ method may lead to worse results than in the case of the TIA and $A O D$ methods $[7,30,31]$.

In summary, this paper shows the advantage of automatic calculations of the minimum distance between the iris and the cornea within a specified range over the other previously known methods. The disadvantages of these known methods are: point measurement, lack of full automation or obtaining a series of information instead of a single number. The new proposed method is free of these defects. It enables to successfully diagnose pathological cases of iridocorneal angle narrowing, which is not possible with other available methods. It also enables to obtain very high repeatability of measurements and record the results in the form of single numbers. Owing to this method, implemented to any OCT device, ophthalmologists will receive more accurate results fully automatically - without any manual intervention. Not only will it increase comfort but it will also give rapid and reproducible results. In addition, it will enable to perform an automatic statistical analysis of a selected population of patients and monitor the progress of treatment, which will directly influence the costs of treatment, prevention and diagnosis of patients.

\section{Abbreviations}

AOD: Angle opening distance; TIA: Trabecular-iris angle; TISA: Trabecular-iris space area; AOS: Angle Opening Sequence; AOM: Angle Opening Minimum; AOM2: Angle Opening Minimum 2; ROC: Receiver Operating Characteristic; ACC: Accuracy; SPC: Specificity; TP: True positive; TN: True negative; FN: False negative; FP: False positive. 


\section{Author contributions}

RK and ZW suggested the algorithm for image analysis and processing, implemented it and analysed the images. SW, AN, EW performed the acquisition of the OCT images and consulted the obtained results. All authors have read and approved the final manuscript.

\section{Acknowledgements}

No outside funding was received for this study.

\section{Author details}

'Department of Biomedical Computer Systems, Institute of Computer Science, University of Silesia, Będzińska 39 Str, Sosnowiec 41-200, Poland. ${ }^{2}$ Department of Biophysics, School of Pharmacy, Medical University of Silesia, Jednosci 8 Str, Sosnowiec 41-200, Poland. ${ }^{3}$ Oddział Okulistyki Okręgowego Szpitala Kolejowego w Katowicach, Okręgowy Szpital Kolejowy w, Katowicach, Poland.

Received: 10 February 2013 Accepted: 6 May 2013

Published: 10 May 2013

\section{References}

1. Ishikawa H, Schuman JS: Anterior segment imaging: ultrasound biomicroscopy. Ophthalmol Clin North Am 2004 7(1):7-20.

2. Kai-shun LC, Li H, Neal WR, Liu J, Yim LC, Yiu KLR, Pui PC, Shun CD: Anterior Chamber Angle Measurement with Anterior Segment Optical Coherence Tomography. Comparison Between Slit Lamp OCT Visante OCT IOVS 2008, 49(8):3469-3474.

3. Wirbelauer C, Karandish A, Häberle H, Thoai D: Pham Noncontact Goniometry With Optical Coherence Tomography. Arch Ophthalmol 2005, 123:179-185.

4. Radhakrishnan S, See J, Smith SD, Nolan WP, Ce Z, Friedman DS, Huang D, Li Y, Aung T, Chew PTK: Reproducibility of Anterior Chamber Angle Measurements Obtained with Anterior Segment Optical Coherence Tomography. IOVS 2007, 48(8):3683-3688

5. Liu S, Li H, Dorairaj S, Cheung CY, Rousso J, Liebmann J, Ritch R, Lam DS, Leung CK: Assessment of scleral spur visibility with anterior segment optical coherence tomography. J Glaucoma 2010, 19(2):132-135.

6. Koprowski R, Wrobel Z, Nowinska A, et al: Automatic Measuring of the Iridocorneal Angle in the Optical Coherence Tomographic Image of the Anterior Segment of the Eye. Inf Technol In Biomed Vol 2 Book Ser Adv Intell Soft Comput 2010, 69:175-182.

7. Koprowski R, Wróbel Z: Image Processing in Optical Coherence Tomography: Using Matlab. Katowice, Poland: University of Silesia; 2011 [http://www.ncbi.nlm.nih.gov/books/NBK97169/].

8. Sakata LM, Lavanya R, Friedman DS, Aung HT, Seah SK, Foster PJ, Aung T: Assessment of the scleral spur in anterior segment optical coherence tomography images. Arch Ophthalmol 2008, 126:18-185.

9. Koprowski R, Wróbel Z: Hierarchic approach in the analysis of tomographic eye image. Comput Recognit Syst 3 Book Ser Adv Intell Soft Comput 2009, 57:463-470.

10. Koprowski R, Wróbel Z: Layers recognition in tomographic eye image based on random contour analysis. Comput Recognit Syst 3 Adv Intell Soft Comput 2009, 57:471-478.

11. Korzynska A, Iwanowski M: Multistage morphological segmentation of bright-field and fluorescent microscopy images. Opto-Electron Rev 2012, 20(2):174-186.

12. Porwik P, Para T: Some handwritten signature parameters in biometric recognition process. In Proceedings of the ITI 2007 29th International Conference on Information Technology Interfaces Book Series. Dubrovnik Croatia: ITI; 2007:185-190.

13. Sonka M, Michael Fitzpatrick J: Medical Image Processing and Analysis. In Handbook of Medical Imaging. Belligham: SPIE; 2000

14. Korzynska A, Hoppe A, Strojny W, et al: Investigation of a combined texture and contour method for segmentation of light microscopy cell images. In Biomedical Engineering: Proceedings of the Second IASTED International Conference. 2004:234-239.

15. Yoo C, Hyun OJ, Yeon KY, Hai RJ: Peripheral Anterior Synechiae and Ultrasound Biomicroscopic Parameters in Angle-Closure Glaucoma Suspects. Kor J Ophthalmol 2007, 21(2):106-110.

16. Kim M, Park KH, Kim TW, Kim DM: Changes in anterior chamber configuration after cataract surgery as measured by anterior segment optical coherence tomography. Korean J Ophthalmol 2011, 25(2):77-83,

17. Furuya T, Mabuchi F, Chiba T, Kogure S, Tsukahara S, Kashiwagi K: Comparison of the anterior ocular segment measurements using swept-source optical coherent tomography and a scanning peripheral anterior chamber depth analyzer. Jpn J Ophthalmol 2011, 55(5):472-479.

18. Liu S, Yu M, Ye C, Lam DS, Leung CK: Anterior chamber angle imaging with swept-source optical coherence tomography: an investigation on variability of angle measurement. Invest Ophthalmol Vis Sci 2011, 52(12):8598-8603.

19. Otsu N: A threshold selection method from gray-level histograms. IEEE Trans Sys Man Cyber 1979, 9(1):62-66.

20. Christopher Wirbelauer MD, Amir Karandish MD, Heike Häberle MD, Duy Thoai Pham MD: Noncontact Goniometry With Optical Coherence Tomography. Arch Ophthalmol 2005, 123(2):179-185.

21. Leung CK, Li H, Weinreb RN, Liu J, Cheung CY, Lai RY, Pang CP, Lam DS: Anterior chamber angle measurement with anterior segment optical coherence tomography: a comparison between slit lamp OCT and Visante OCT. Invest Ophthalmol Vis Sci 2008, 49(8):3469-3474.

22. Ang GS, Wells AP: Changes in Caucasian eyes after laser peripheral iridotomy: an anterior segment optical coherence tomography study. Clin Experiment Ophthalmol 2010, 38(8):778-785.

23. Dada T, Sihota R, Gadia R, Aggarwal A, Mandal S, Gupta V: Comparison of anterior segment optical coherence tomography and ultrasound biomicroscopy for assessment of the anterior segment. J Cataract Refract Surg 2007, 33:837-840, 
24. Kalev-Landoy M, Day AC, Cordeiro MF, Migdal C: Optical coherence tomography in anterior segment imaging. Acta Ophthalmol Scand 2007, 85(4):427-430.

25. Sakata LM, Lavanya R, Friedman DS, Aung HT, Seah SK, Foster PJ, Aung T: Assessment of the scleral spur in anterior segment optical coherence tomography images. Arch Ophthalmol 2008, 126(2):181-185.

26. Xu L, Cao WF, Wang YX, Chen CX, Jonas JB: Anterior Chamber Depth and Chamber Angle and Their Associations with Ocular and General Parameters. The Beijing Eye Study. Am J Ophthalmol 2008, 145(5):929-936.

27. Baikoff G, Lutun E, Ferraz C, Wei J: Static and dynamic of the Anterior Segment with Optical Coherence Tomography. J Cataract Refract Surg 2004, 30(9):1843-1850.

28. Phillips Cl: Closed-iridocorneal angle: significance of sectorial variations in angle depth. Br J Ophthalmol 1956, 40:136-143.

29. Dorairaj SK, Tello C, Liebmann JM, et al: Narrow angles and angle closure: anatomic reasons for earlier closure of the superior portion of the iridocorneal angle. Arch Ophthalmol 2007, 125:734-739.

30. Muftuoglu O, Hosal BM, Zilelioglu G: Ciliary body thickness in unilateral high axial myopia. Eye (Lond) 2009, 23:1176-1181.

31. Oliveira C, Tello C, Liebmann JM, et al: Ciliary body thickness increases with increasing axial myopia. Am J Ophthalmol 2005, 140:324-325.

doi:10.1186/1475-925X-12-40

Cite this article as: Koprowski et al:: Methods of measuring the iridocorneal angle in tomographic images of the anterior segment of the eye. BioMedical Engineering OnLine 2013 12:40.

\section{Submit your next manuscript to BioMed Central and take full advantage of:}

- Convenient online submission

- Thorough peer review

- No space constraints or color figure charges

- Immediate publication on acceptance

- Inclusion in PubMed, CAS, Scopus and Google Scholar

- Research which is freely available for redistribution

Submit your manuscript at www.biomedcentral.com/submit 